\title{
Individual Health Literacy Assets
}

Janine Bröder, Orkan Okan, Torsten M. Bollweg, Dirk Bruland, Paulo Pinheiro, Ullrich Bauer

\section{Source}

Janine Bröder, Orkan Okan, Torsten M. Bollweg, Dirk Bruland, Paulo Pinheiro, Ullrich Bauer. (2019). Child and Youth Health Literacy: A Conceptual Analysis and Proposed Target-Group-Centred Definition. IJERPH, vol. 16 (18), 3417.

Namely, the personal cog nitive and habitual characteristics/attributes including the child's independent knowledge along with abilities such as the ability to change, belief systems, cultural norms, and motivations. 